\title{
Educación superior inclusiva: Un reto para las prácticas pedagógicas
}

\author{
Inclusive Higher Education: A Challenge for Pedagogical Practices
}

\section{Ensino Superior inclusivo: um desafio para as práticas pedagógicas}

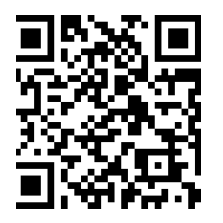

Ruth Zárate-Rueda ${ }^{1}$

Universidad Industrial de Santander

Bucaramanga, Santander, Colombia

ruthzaraterueda@gmail.com

http://orcid.org/000-0001-8060-8777

Sonia Patricia Díaz-Orozco 2

Universidad Autónoma de Bucaramanga

Bucaramanga, Santander, Colombia

sdiaz682@unab.edu.co

http://orcid.org/000-0002-3658-3696

Leonardo Ortiz-Guzmán ${ }^{3}$

Universidad Industrial de Santander

Bucaramanga, Santander, Colombia

leortizguz@gmail.com

http://orcid.org/0000-0002-4530-8039

Recibido 30 de mayo de 2016 • Corregido 1 de agosto de 2017 • Aceptado 25 de agosto de 2017

Received 30 de mayo de 2016 • Revised 1 de agosto de 2017 • Accepted 25 de agosto de 2017

Recebido 30 de mayo • Revisado 1 de agosto de 2017 • Aprovado 25 de agosto de 2017

\begin{abstract}
${ }^{1}$ Trabajadora Social de la Universidad Industrial de Santander (UIS); Doctora en Educación de la UPEL (Venezuela); especialista en: investigación social, docencia universitaria, educación sexual y procesos afectivos; magíster en evaluación en educación. Ha sido Directora de Investigación y Extensión de la Facultad de Ciencias Humanas (UIS); profesora titular de la Escuela de Trabajo Social, UIS; directora línea Responsabilidad e innovación social, grupo de investigación INNOTEC UIS.

${ }^{2}$ Administradora de Empresas, UNIBOYACÁ. Especialista en Necesidades Educativas e Inclusivas (NEEI), Universidad Autónoma de Bucaramanga (UNAB). Magistra en Educación, UNAB. Profesora pregrado y postgrado en NEEl. Docente apoyo para educación inclusiva. Colegio ASPAEN Gimnasio Cantillana.

${ }^{3}$ Licenciado en Español y Literatura de la Universidad Industrial de Santander (UIS); Especialista en Necesidades Educativas e Inclusión-UNAB. Estudiante Maestría en Educación (UNAB). Docente de literatura, Gimnasio San Diego (Floridablanca, Santander) e investigador social en la línea Responsabilidad e innovación social, grupo de investigación INNOTEC UIS.
\end{abstract}


doi: http://dx.doi.org/10.15359/ree.21-3.15

URL: http://www.una.ac.cr/educare

CORREO: educare@una.cr

Resumen: El presente artículo científico muestra los resultados de un proceso de investigación que tuvo como objetivo analizar las prácticas y estrategias pedagógicas que implementan docentes de la Universidad Industrial de Santander (UIS) y la Universidad Autónoma de Bucaramanga (UNAB), quienes cuentan en sus aulas con estudiantes con discapacidad sensorial. Para este fin, se optó por una metodología cualitativa con un enfoque de estudios de casos en el cual se participaron 27 sujetos: 20 docentes y 7 estudiantes de los programas de las escuelas de Derecho, Negocios Internacionales, Contaduría, Comunicación Social, Gastronomía, Música y Filosofía. A partir de un muestreo de casos homogéneos de tipo intencional y entrevistas semiestructuradas, se pudo concluir que, a pesar de los avances significativos dados hasta el momento, se requiere que las universidades promuevan lineamientos institucionales que articulen el trabajo participativo con la comunidad educativa que interviene en la realidad social del estudiantado con discapacidad, así como en los ejes de accesibilidad y comunicación.

Palabras claves: Educación integradora; formación profesional superior; método de enseñanza.

Abstract: This scientific article shows the results of a research process whose objective was to analyze the practices and pedagogical strategies implemented by teachers of the Industrial University of Santander (UIS) and the Autonomous University of Bucaramanga (UNAB); these teachers have students with sensory disabilities in their classrooms. For this goal, a qualitative methodology was adopted with a case-study approach; 27 subjects participated in this process: 20 educators and 7 students from the programs of Law, International Business, Accounting, Social Communication, Gastronomy, Music, and Philosophy. Based on a sample of homogenous cases of intentional type and semi-structured interviews, it was possible to conclude that, despite the significant advances made so far, it is required for universities to promote institutional guidelines articulating participatory work with the educational community. This community intervenes in the social reality of students with disabilities, as well as in the axes of accessibility and communication.

Keywords: Inclusive education; higher vocational education; teaching method.

Resumo: Este artigo científico mostra os resultados de um processo de pesquisa que visa analisar as práticas e estratégias pedagógicas implementadas pelos professores da Universidade Industrial de Santander (UIS) e da Universidade Autônoma de Bucaramanga (UNAB), que têm em sua salas de aula estudantes com deficiências sensoriais. Para este fim, opta-se por uma metodologia qualitativa focada em estudos de caso proporcionados por 27 pessoas participantes: 20 professores e 7 estudantes dos cursos de Direito, Negócios Internacionais, Contabilidade, Comunicação Social, Gastronomia, Música e Filosofia. A partir de uma amostragem de casos homogêneos de tipo intencional e semiestruturado, concluiu-se que, apesar dos progressos significativos realizados até agora, as universidades devem promover diretrizes institucionais capazes de articular o trabalho participativo com a comunidade educativa envolvida na realidade social dos estudantes com deficiência, bem como os princípios de acessibilidade e comunicação.

Palavras-chave: Educação inclusiva; formação profissional superior; método de ensino. 


\section{Introducción}

Las personas con discapacidad constituyen un grupo minoritario segregado en diversas áreas sociales, como el sistema educativo: desde la primera infancia hasta los niveles de educación superior esta población carece de condiciones para la participación y procedimientos que garanticen el acceso y permanencia en igualdad de condiciones con sus pares sin discapacidad. En Colombia, al igual que en diferentes países, desde la década de los noventa la inclusión educativa se ha convertido en tema de relevancia social; de ahí que especialistas en el tema, como Blanco (2008), la definen como el proceso de identificar y responder a la diversidad de necesidades de todo el estudiantado, a través de la mayor participación en el aprendizaje, las culturas y las comunidades, para lo cual es necesario desarrollar ajustes en lo referente a la pedagogía, contenido, enfoques y estrategias que conlleven a facilitar el acceso a la educación de personas con discapacidad sensorial sin relegar al resto de la población.

Si bien es cierto que en Colombia la carta magna de 1991 plantea un marco constitucional político que aduce la educación como un derecho inalienable de toda la ciudadanía que debe asegurarse a lo largo de su formación (Asamblea Nacional Constituyente, 1991), otros referentes legales como la Ley 30 de diciembre 28 de 1992, por el cual se organiza el servicio público de la educación superior (Congreso de Colombia, 1992), y la Ley Estatutaria 1618 de 2013; se establecen las disposiciones para garantizar el pleno ejercicio de los derechos de las personas con discapacidad (Congreso de Colombia, 2013), es una realidad que en el contexto universitario se adolece de rutas de acompañamiento y programas de apoyo que aseguren a este estudiantado su ingreso, permanencia y culminación satisfactoria, lo cual impide que se dé una garantía y un goce efectivo de los derechos de la población con necesidades educativas especiales e inclusivas -NEEI-.

Por lo expuesto anteriormente, es imperativo que desde el sector educativo se asuma una mayor responsabilidad en la creación de procesos innovadores que garanticen a las personas con discapacidad el acceso al sistema formativo. En consecuencia, las instituciones de educación superior-IES deben desarrollar, dentro de sus políticas educativas, prácticas inclusivas mediante la cualificación de sus docentes que les permitan desarrollar pedagogías flexibles que integren a la diversidad del estudiantado.

Dentro de este contexto, la investigación se planteó como propósito analizar las prácticas pedagógicas queimplementan los docentes ylas docentes en dos universidades de Bucaramanga (Santander-Colombia): la Universidad Industrial de Santander, UIS y la Universidad Autónoma de Bucaramanga, UNAB. La primera de ellas es una institución pública de carácter oficial, líder en el nororiente colombiano con reconocimiento de alta calidad, orientada a la construcción integral del ser y difusión del conocimiento mediante las siguientes ramas del conocimiento: ingeniería físico-mecánicas, ingenierías fisicoquímicas, ciencias, salud y humanidades. Estas, a su vez, conjugan los campos de conocimiento en actividades que constituyen las funciones misionales 
doi: http://dx.doi.org/10.15359/ree.21-3.15

URL: http://www.una.ac.cr/educare

CORREO: educare@una.cr

de docencia, investigación y extensión. La segunda se caracteriza por ser la primera institución de carácter privado del oriente colombiano en lograr la acreditación de alta calidad. Al servicio de la educación superior, su propósito de engrandecimiento del ser humano se traduce en los principios democráticos y liberales que guían su acción, propendiendo al mejoramiento de la calidad de educación regional y nacional.

Las dos universidades fueron seleccionadas debido a que desde sus aulas se forman estudiantes con discapacidad sensorial en distintas áreas del conocimiento; es justamente allí donde estriba la intencionalidad de la investigación, ya que se busca dimensionar el compromiso frente a la educación inclusiva desde el ámbito universitario. Precisamente, los hallazgos derivados del estudio se constituirán en fundamentos que permitan plantear alternativas y estrategias pedagógicas que cualifiquen la praxis del personal docente y faciliten procesos de adaptación a estudiantes con estas características. Para finalizar, es importante mencionar que el análisis de las prácticas de estas dos universidades como sujetos de investigación no consiste en una comparación entre sí, ya que la intención de este estudio es ampliar las fuentes de información de manera que permitan la construcción de saberes sólidos que repercutan en la labor docente a mediano plazo.

\section{Argumentación teórica}

Para comprender los referentes que engloban a la inclusión educativa, es pertinente detallar en qué consisten estos procesos de aprendizaje en la educación superior, destacando los avances que se han establecido en las IES para garantizar la permanencia de estudiantes con necesidades educativas especiales e inclusivas (NEEI) en sus aulas. A continuación, se definen los aspectos más relevantes de la inclusión.

\section{Definiciones de discapacidad}

A lo largo de la historia, la discapacidad ha estado vinculada a sentidos negativos por cuanto se calificaba a la persona como dañada -física, sensorial o intelectualmente- en comparación con el estado inicial del sujeto, así como en términos de producción económica (Vásquez y Cáceres, 2008). Asimismo, la Organización Mundial de la Salud (OMS, 2001) concibe la discapacidad como un término general que abarca las deficiencias, limitaciones de la actividad y las restricciones de la participación.

Según este organismo, las deficiencias son problemas que afectan una estructura o función corporal; las limitaciones de la actividad son dificultades para ejecutar acciones o tareas, y las restricciones de la participación son los diversos problemas para participar en situaciones vitales. Por consiguiente, la discapacidad es un fenómeno complejo que refleja interacción entrelas características del organismo humano y las particularidades del contexto en el que la persona está inmersa. 
Por otra parte, Zárate (2007, citado por Zárate y Rodríguez, 2014) sostiene:

La discapacidad como una realidad humana, ha sido considerada en el transcurrir del tiempo, de diferentes modos correspondiendo la mayoría de las concepciones a las condiciones de la persona, desconociendo factores del entorno como la limitante en sus actuaciones. Para algunos la discapacidad significa una condición patológica; pero si bien, esta se puede originar en una enfermedad, se debe entender como una consecuencia y no la enfermedad en sí. En el campo de la salud, también se concibe la discapacidad como el fenómeno complejo que refleja una interacción entre las características del organismo humano y las características de la sociedad en la que vive. (p. 11)

Desde este planteamiento, se logra identificar que a través de la historia, solo quienes aportan económicamente al desarrollo social y político son partícipes en el sistema social instaurado: se margina a esta población, con base en dicha perspectiva errónea, a tal punto que se restringe su preparación mediante la inatención educativa y laboral para la participación social. No obstante, a partir de la perspectiva social, se considera que las personas en condición de discapacidad son actores sociales que igualmente aportan a la sociedad desde sus posibilidades (Palacios, 2008).

Así pues, y con una estrecha relación con los valores de los derechos humanos, a nivel educativo la discapacidad desde el enfoque social implica una inclusión educativa: estrategias flexibles que reduzcan las barreras en el aprendizaje del estudiantado, de manera que adquiera las habilidades requeridas para una labor independiente, así como la participación social. Por esta razón, a partir de la perspectiva social se incluye la vivencia de experiencias particulares y significativas de su realidad que involucren la alteración funcional, con lo estructural y las oportunidades ofrecidas por el entorno.

Finalmente, la diversidad funcional pretende ser la síntesis de un conjunto de ideas sistemáticamente organizado para la comprensión de una realidad social por lo común denominada como "discapacidad" y lo hace con clara pretensión emancipadora, para evitar mantener la exclusión y marginación propia de este concepto, vivida por las personas que poseen algún tipo de limitación (Rodríguez y Ferreira, 2010). Para el caso de esta investigación se aborda el término discapacidad sensorial, el cual se constituye como un grupo de personas con deficiencias visuales y auditivas, las cuales presentan dificultades en la comunicación y el lenguaje.

\section{Fundamentos de la inclusión y la educación inclusiva}

En el mundo globalizado el acceso a la información, formación y actividad laboral adquiere importancia para alcanzar niveles óptimos de calidad de vida: la formación y capacitación son la principal estrategia que permite tal fin, a partir de la eliminación de barreras y garantía del acceso 
doi: http://dx.doi.org/10.15359/ree.21-3.15

URL: http://www.una.ac.cr/educare

CORREO: educare@una.cr

y la promoción educativa a lo largo de la vida, de acuerdo con lo señalado en el apartado anterior. Así pues, se requiere alejarse del confuso concepto de inclusión escolar, el cual se limita solo al ingreso del estudiantado al aula de clase sin la flexibilización y atención requerida; para dirigirse a la implementación de un enfoque de una educación para todos y todas o educación inclusiva, en el cual se favorezca el desarrollo educativo y profesional de personas en situación de discapacidad.

Bayliss, Van Hove, Windels, Carro y Hinz (1997) señalan que la inclusión tiene que ver con todas las personas, los niños, niñas, mujeres, ancianos, ancianas, hombres, pobres, enfermos y enfermas, quienes tienen discapacidad; así mismo, tiene que ver con las personas ricas, los sanas y capaces. El rechazo de las diferencias individuales, según Ainscow (2001), es uno de los factores generadores de exclusión, debido a que se considera que son aspectos de experiencia común y, por tanto, todo el estudiantado puede presentar dificultades de aprendizaje que, en diferentes ocasiones, son resultado de la interacción escuela-estudiante.

En este enfoque, las características particulares de cada persona deben ser el punto de partida del personal educador para desarrollar al máximo su potencial, lo cual exige dirigir una especial atención a la respuesta del estudiantado. Como lo afirma Ortiz (2000), el primer paso hacia la escuela inclusiva es la aceptación incondicional de las diferencias, ya que la diversidad fortalece al alumnado al ofrecerle alternativas de aprendizaje. En efecto, en la comprensión de la enseñanza es donde se percibe lo complejo de la acción docente, por lo que para garantizar lo anterior, este autor sugiere adoptar una propuesta interactiva de enseñanza-aprendizaje. Estos preceptos están relacionados con la calidad de la educación, por lo cual es importante que las IES garanticen y generen constantemente apoyo y recursos para la escuela inclusiva.

Ahora bien, Barton y Armstrong (2001) entienden la inclusión como un proyecto social en donde la educación es el camino para conseguir sociedades más justas: la educación inclusiva no es un propósito en sí misma, sino un medio para alcanzar un fin, en este caso construir una sociedad equitativa. Estos procesos de integración educativa se han fortalecido a través de movimientos sociales que han generado un nuevo paradigma que insiste en el "todos" $y$ "para todos". Por tanto, como nueva lucha contra cualquier tipo de exclusión, se pretende establecer una escuela y sociedad en la que no se excluya a ningún tipo de personas (Andrés y Sarto, 2009).

La educación inclusiva, según Blanco (2008), hace referencia a un proceso que entraña la transformación de la academia y otros centros de formación para atender a la población con determinadas características, tales como: los niños y las niñas, las minorías étnicas, personas con necesidades educativas especiales e inclusivas (NEEI), con discapacidad o dificultades de aprendizaje, habitantes de fronteras, grupos desmovilizados, víctimas de la violencia y a personas afectadas por el VIH/SIDA. El objetivo, ofrecer una educación de calidad respetando la diversidad, las distintas necesidades, aptitudes, características y expectativas tanto de estudiantes como de las comunidades y su entorno, eliminando con esto toda forma de discriminación. 
Las autoras Gutiérrez, Martín y Jenaro (2014, p. 187), especialistas en inclusión social, sostienen:

La inclusión tiene que ver con el proceso de incrementar y mantener la participación de todas las personas en la sociedad, escuela o comunidad de forma simultánea, procurando disminuir y eliminar todo tipo de procesos que lleven a la exclusión (Booth, 1996). Por ello, un centro inclusivo se caracterizará, tal y como señala Ainscow (1999), por la ausencia de toda forma de discriminación en él, cuestiones tales como la justicia social, la equidad, los derechos humanos y la no discriminación serán sus elementos clave (Barton, 2009).

El acceso a una educación participativa y el relacionarse con sus pares permite un aprendizaje de calidad en el estudiantado (Blanco, 2008), especialmente en quienes tienen necesidades educativas derivadas de discapacidades. No obstante, la inclusión resalta el proceso de identificar y responder a la diversidad de sus necesidades, a través de una interacción consciente en el aprendizaje, las culturas y comunidades, reduciendo la exclusión en la educación (Rosano, 2007). De la Oliva (2007) reconoce que la integración educativa logró situar en la escuela la mayor parte de los problemas, dando paso al replanteamiento de los objetivos académicos, involucrando también cambios y modificaciones en contenidos, aproximaciones, estructuras y una transformación profunda soportada en la reforma (Velázquez, 2010).

\section{Prácticas pedagógicas, educativas e inclusivas: La educación inclusiva como estrategia de inclusión social}

Zaccagnini (2008) sostiene que en la interacción social entre un sujeto mediador (sujeto pedagógico) con otro (sujeto educando), denominadas prácticas pedagógicas, surgen acciones educativas complejas orientadas al cumplimiento de unos objetivos establecidos por el primero. Empero, estas con dificultad pueden ser generalizadas teniendo en cuenta que no todos los sujetos poseen las mismas habilidades. Por ejemplo, la población con algún tipo de discapacidad (física, sensorial o cognitiva) requiere estrategias dirigidas a satisfacer las necesidades dentro y fuera del aula de clase: modelos flexibles de enseñanza y evaluación de aprendizajes, así como rutas de atención y apoyo que promuevan la permanencia en la educación superior y su culminación satisfactoria.

La práctica educativa la ejerce una institución que planifica, organiza y fortalece el proceso educativo, con el ánimo de transformarse en una práctica efectiva y coherente con los propósitos universitarios. Su función es clara al ser relacionada con las estructuras sociales, con la academia, con sus planes, programas, teorías, acciones y con el contexto social en el que se desenvuelve. Como praxis deliberada persigue fines explícitos, por tanto, "la reflexión crítica sobre la práctica se torna una exigencia de la relación Teoría/Práctica sin la cual la teoría puede convertirse en palabrería y la práctica en activismo" (Freire, 2004, p. 24). De lo anterior, se aduce que la práctica docente es un proceso dinámico y reflexivo que comprende los acontecimientos ocurridos en la interacción entre sujeto educador-sujeto educando. 
doi: http://dx.doi.org/10.15359/ree.21-3.15

URL: http://www.una.ac.cr/educare

CORREO: educare@una.cr

Coll (1996) señala que la actividad del profesorado tiene una estrecha relación con la manera cómo aprenden y se desarrollan el estudiantado, con las dificultades y problemas que se encuentran cuando lleva a cabo nuevos aprendizajes y con las intervenciones dirigidas a apoyarle para que supere dichas dificultades. El personal docente participa en el análisis, planificación, desarrollo y la modificación de los procesos educativos; por tanto, deben formarse para desarrollar una serie de competencias que le permitan ser inclusivo como parte de su accionar profesional (Fernández, 2012).

Los planteamientos del Ministerio de Educación Nacional (MEN, 2007) aportan a la comprensión del proceso de educación inclusiva en la educación superior una serie de momentos que permiten comprender la ruta de atención como parte de su labor e impacto a nivel social y académico; es así como, desde este ministerio, se propone una serie de estrategias para la implementación en ambientes académicos superiores e incluyentes:

En primer lugar, inicia en el 2007 con un estudio realizado por el Centro de Investigación para el Desarrollo (CID), segundo, entre 2007 y 2011 adopta un enfoque poblacional que transita de necesidades especiales educativas (NEE) a necesidades educativas diversas (NED). En tercer orden, en 2012 desarrolla un estudio conceptual en el cual se desvirtúa la NED por educación inclusiva (El); una vez definida y caracterizada la El para el contexto colombiano, en el cuarto momento, se priorizan los grupos más proclives a ser excluidos del sistema, desde la visión de la política diferencial. Asimismo, como quinto punto lleva a cabo entre 2012 y 2013 la convocatoria de programas de inclusión, para luego establecer la denominada "Educación para todos", como objetivo final en educación hasta el 2026. Posteriormente, como última estrategia implementa la fase: "sociedades incluyentes", a largo plazo dado que se proyecta hasta el año 2034.

Según Ainscow (2001), la educación inclusiva tiene seis características producto de la reflexión conceptual de las diferentes definiciones que existen: participación, diversidad, interculturalidad, equidad, pertinencia y calidad. Con base en estos planteamientos y desde las orientaciones del Ministerio de Educación Superior en Colombia, se ratifican los preceptos establecidos para la educación incluyente, con el objeto de facilitar el acceso a las IES para todas las personas, sin ningún tipo de exclusión. En este sentido, es fundamental precisar que, para generar procesos reales de educación inclusiva, las IES tienen como reto engranar las anteriores características e incorporarlas en la implementación de estrategias que garanticen el acceso a todas las personas con discapacidad, desde el enfoque de los derechos humanos, el cual tiene como núcleo el respeto y fomento de la diversidad e interculturalidad con equidad, calidad y participación en los sistemas educativos.

\section{Metodología}

Se determinó desarrollar una investigación cualitativa pues, de acuerdo con Hernández, Fernández y Baptista (2010), se estudia la calidad de las actividades, relaciones, asuntos, medios, 
materiales o instrumentos en una determinada situación o problema: Por tanto, la descripción de las cualidades de un fenómeno se centra en el entendimiento en profundidad, más que en la exactitud. Desde otra perspectiva, expresa Sandoval (1996) que este tipo de investigación:

Reivindica el abordaje de las realidades subjetiva e intersubjetiva como objetos legítimos de conocimiento científico; el estudio de la vida cotidiana como el escenario básico de construcción, constitución y desarrollo de los distintos planos que configuran e integran las dimensiones específicas del mundo humano y, por último, ponen de relieve el carácter único, multifacético y dinámico de las realidades humanas. (p. 15)

Para comprender en profundidad la realidad social y educativa, el método empleado fue el estudio de caso, según Stake (1998), es una descripción y análisis detallado de unidades sociales, en particularidad y de la complejidad de una realidad singular, para llegar a interpretar sus acciones en escenarios específicos y concretos. Una característica de este método es el estudio intensivo y profundo de unos casos o una situación con cierta intensidad. Este es relevante en el desarrollo de las ciencias sociales y humanas, representando la forma más pertinente y natural de las investigaciones orientadas desde una perspectiva cualitativa (Latorre, Del Rincón \& Arnal, 1996), pues permite a las investigaciones captar el conocimiento, el significado y las interpretaciones que comparten los individuos sobre la realidad social estudiadA. En efecto, "su mayor fortaleza radica en que a través del mismo se mide y registra la conducta de las personas involucradas en el fenómeno estudiado" (Martínez, 2006, p. 167).

Finalmente, y de acuerdo con Stake (1998), el estudio de caso puede apoyarse desde la teoría fundamentada, la hermenéutica o la etnografía de acuerdo con los objetivos propuestos. Para esta investigación, se orientó desde la etnografía por cuanto se pretende profundizar en la cultura pedagógica establecida en las instituciones universitarias que desarrollan docentes en la formación de estudiantes con discapacidad sensorial, en la UIS y la UNAB, con miras a la definición de estrategias que contribuyan a la consolidación de instituciones socialmente responsables e incluyentes: roles, acciones, significación de contextos, entre otros.

\section{Actores sociales y fases metodológicas}

La población estuvo constituida por un total de 27 sujetos de la comunidad educativa de dos instituciones de educación superior (pública -26.000 estudiantes-- y privada -14.000 estudiantes-): 20 personas educadores que abordan desde el acto pedagógico a estudiantes que presentan una condición de discapacidad sensorial (visual o auditiva) y, 7 estudiantes con discapacidad sensorial de los programas de Derecho, Negocios Internacionales, Contaduría, Comunicación Social, Gastronomía, Música y Filosofía. Se implementó el muestreo de casos homogéneos de tipo intencional que, según Sandoval (1996), se caracteriza por la identificación de sujetos con experiencias comunes sobre el tema de investigación. 
doi: http://dx.doi.org/10.15359/ree.21-3.15

URL: http://www.una.ac.cr/educare

CORREO: educare@una.cr

El proceso metodológico se desarrolló en cuatro fases que permitieron la comprensión de las prácticas pedagógicas en ambientes educativos. Estas se enuncian a continuación:

Revisión documental: Una vez identificado el problema de investigación, concerniente a la falta de rutas y programas de apoyo que asegure al estudiantado con discapacidad su ingreso, permanencia y culminación satisfactoria de su formación superior; se procedió a construir el estado del arte entendido como un estudio analítico del conocimiento acumulado. Su propósito fue sistematizar la producción del área de conocimiento que permitió hacer una reflexión sobre tendencias y vacíos en la temática (Vargas y Calvo, 1987); así como determinar los fundamentos teóricos y legales conforme a las previas categorías investigativas. En otras palabras, un proceso de precategorización que orientó conceptualmente la fase de campo.

Fase de campo: Se realizó directamente en el campus de cada universidad en escenarios donde se llevan a cabo las prácticas que el personal docente implementa en el aprendizaje del estudiantado con discapacidad sensorial, ya sea visual o auditiva. A partir de la precategorización de la fase anterior se procedió a la construcción de las entrevistas semiestructuradas con preguntas abiertas que giraron alrededor de: presaberes sobre necesidades educativas e inclusión, estrategias y evaluaciones flexibles, conocimiento de programas institucionales sobre discapacidad, etc.

De manera complementaria, se utilizó la observación de campo de forma descriptiva en los espacios donde transcurre la cotidianidad de los actores del acto pedagógico, consignando anotaciones según una guía establecida, a partir de la testificación de los relatos, situación que permitió validar los datos recolectados en las entrevistas dirigidas a los sujetos.

Análisis de resultados: en la investigación cualitativa es necesario que los hallazgos estén respaldados por un registro sistemático. Por tanto, la información recolectada pasó inicialmente por un proceso de codificación y reducción de información cuyo criterio de selección fueron los objetivos de investigación. Posteriormente, a partir de lectura continua e interpretación de datos recolectados, los cuales se organizaron y registraron de manera permanente, emergieron las categorías de análisis que brindaron la posibilidad de hacer especificaciones propias del proceso pedagógico.

Lo anterior permitió que el personal docente participante en este estudio, testificaran las prácticas pedagógicas que, desde su punto de vista, facilitan el aprendizaje al estudiantado. De este modo se interpretó el derecho que tienen las personas con discapacidad de acceder a la educación en igualdad de condiciones a la de sus pares sin discapacidad, desde el concepto de equiparación de oportunidades y así dar respuesta a la pregunta orientadora del estudio de caso.

Esta fase se complementó desde el ejercicio de triangulación de la información y se estructuró a partir de los siguientes aspectos: 
1. La realidad educativa, en la que se encuentran las personas, docentes y estudiantes con sus diferentes percepciones, experiencias y reflexiones consolidadas en la construcción escrituraria desprendida de las aserciones de los sujetos participantes en los procesos de aprendizaje con necesidades educativas especiales e inclusivas (NEEI).

2. La teoría, desde los referentes teóricos conceptuales se construyeron las formas de profundización, hacia la comprensión del sujeto de estudio.

3. Observador, sujeto investigador quien al situarse desde el horizonte histórico como portador de experiencias, prejuicios y saberes le da sentido y significado a los resultados recopilados, organizándose en las categorías emergentes para una mejor expresión narrativa metodológicamente hablando.

\section{Análisis y discusión de resultados}

Esta etapa se desarrolló en el marco del objetivo de investigación, el cual consistió en el análisis de las prácticas pedagógicas que implementa el personal docente en la UIS y la UNAB con estudiantes que cuentan con NEEl. Para evidenciar los hallazgos, se realizó el proceso de categorización (categorías y subcategorías) derivado del análisis de los datos. Con el fin de comprender los significados, se emplearon dos estrategias de interpretación: de forma directa y de forma categórica. Esto dio origen al surgimiento de dos categorías centrales: acceso a la educación superior y permanencia de estudiantes. Cabe aclarar que tales categorías coinciden con los lineamientos básicos de educación inclusiva del MEN. En la Tabla 1 se observan las categorías y correspondientes subcategorías establecidas en el proceso investigativo.

Tabla 1: Categorías de investigación

\begin{tabular}{ll}
\hline \multicolumn{1}{c}{ Categoría } & \multicolumn{1}{c}{ Subcategorías } \\
\hline Acceso a la educación superior & Proceso de admisión \\
& Información sobre inclusión de estudiantes \\
& Atención pedagógica a estudiantes con discapacidad \\
\hline Permanencia de estudiantes & Planificación de la enseñanza \\
& Rutinas de clase \\
& Estrategias pedagógicas \\
& Materiales didácticos \\
& Evaluación \\
& Experiencias significativas \\
& Discriminación \\
\hline
\end{tabular}

Nota: Procesamiento de información a partir del trabajo de campo. 
doi: http://dx.doi.org/10.15359/ree.21-3.15

URL: http://www.una.ac.cr/educare

CORREO: educare@una.cr

\section{Categoría 1. Acceso a la educación superior}

\section{Subcategoría 1. Proceso de admisión}

Esta categoría surgió al interrogarse a los sujetos participantes sobre la existencia de un filtro que permita identificar al estudiantado con discapacidad, en el momento de ingreso a las universidades. Las personas entrevistadas expresaron que debe ser responsabilidad de Bienestar Universitario diseñar un protocolo de identificación y abordaje de este tipo de situaciones para que el estudiantado con discapacidad sensorial sea y se sienta incluido dentro de las políticas educativas de cada universidad.

Algunos informantes coincidieron al afirmar que "generalmente en los formularios casi siempre viene la pregunta de si tiene usted alguna discapacidad, señálela" (P1); consideran que la información no es socializada, pues sostienen: "no tengo idea" (P3), "Realmente no lo conozco", "No sé, nunca nos pasan esa información" (P7). A pesar de aplicarse en algunos casos el filtro, tal como lo afirmó el personal docente, desconocen la existencia de políticas, programas y estrategias institucionales que garanticen procesos de acompañamiento, establezcan rutas de abordaje y apoyos diferenciales centrados en las personas, de manera que garanticen la adaptación al ambiente universitario de la población con discapacidad.

Se evidencia, entonces, que aún las universidades no han establecido a cabalidad las acciones pedagógicas y terapéuticas que posibiliten la inclusión educativa y social para personas con diversidad de limitaciones, consignada en la Ley 30 de diciembre 28 de 1992, por la cual se organiza el servicio público de la educación superior (Congreso de Colombia, 1992).

\section{Subcategoría 2. Información sobre inclusión de estudiantes}

Esta subcategoría emerge con una dicotomía de opiniones alrededor de la inclusión de estudiantes propiamente en escenarios pedagógicos. Por un lado, aunque el personal docente sostiene que sí fue informado del ingreso de un estudiante con discapacidad a su asignatura, no sienten un apoyo en praxis:

Sí, fui informada antes de iniciar nuestro primer semestre del 2015 y además nos invitaron a los docentes a una reunión precisamente para hablar sobre estudiantes con alguna diversidad funcional que ingresaban a diferentes carreras, pero no hubo acompañamiento (P6)

Me sentí sola en mi ejercicio pedagógico, la universidad dice una cosa y hace otra. Bienestar universitario no le invierte en programas ni en profesionales de apoyo, tampoco nos capacitan o nos forman para los procesos de enseñanza con personas con necesidades especiales (P2) 
Esta ausencia pedagógica ubica en un plano tenso al personal docente, por cuanto el ejercicio de desarrollo de habilidades se dirige desde un desconocimiento de la interacción pertinente para la significación de los objetos de conocimiento, que genera algunas dificultades adicionales al estudiante con discapacidad.

Adicionalmente, algunas personas docentes afirmaron desconocer que un estudiante en esta condición estuviera en su aula de clase:

Nunca fui advertido, lo que al principio dificultó las cosas, ya ahora el asunto está superado. Me ha tocado improvisar y hasta inventar, pero todo ha sido de buena voluntad. He consultado experiencias en otras universidades y preguntar a mis compañeros si han tenido una experiencia similar. Pero la situación no es fácil (P12)

En este sentido, se afirma que la información queda guardada en las respectivas oficinas de admisiones y se excluye al personal docente, principal mediador del aprendizaje, de datos requeridos para satisfacer las necesidades académicas de esta población. Ante esto, Blanco (2008) sostiene que el ocultamiento de una información de valor como la señalada conlleva a que el sistema educativo difícilmente atienda a esta población satisfactoriamente: el currículo permanece con un enfoque homogéneo que se aleja de modelos educativos flexibles que consideren la diversidad funcional.

Desde esta perspectiva, se hace necesario que los docentes y las docentes universitarias conozcan con antelación que tendrán un o una estudiante con discapacidad y preparen sus clases teniendo en cuenta estrategias flexibles y pertinentes para la equidad de las necesidades comunes y específicas que estas poblaciones presentan. Para lograr lo anterior, es oportuno que el sistema educativo defina y aplique concepciones éticas que permitan considerar la inclusión como un asunto de derechos humanos, de manera que se implementen procesos de enseñanza-aprendizaje flexibles e innovadores que respondan a una educación con estilos pedagógicos y capacidades diferentes entre el estudiantado, alternativas de acceso al conocimiento y diversidad en evaluaciones de competencia (MEN, 2008).

\section{Subcategoría 3. Atención pedagógica de estudiantes con discapacidad}

Esta categoría es la apreciación directa de las respuestas dadas por el personal docente, en cuanto a las acciones que realiza o estrategias utilizadas cuando conoce que en su materia habrá estudiantes con discapacidad sensorial.

Los sujetos participantes sostuvieron que realizan acciones concretas como: "organizar el currículo y sobre todo hacer ajustes en el plan de estudios para que el aprendizaje sea el más acertado y estos chicos se sientan bien" (P12), "El diálogo con la persona y a partir del diálogo sé que tengo que diseñar nuevas metodologías, porque además debe ser distinto una persona sorda, 
doi: http://dx.doi.org/10.15359/ree.21-3.15

URL: http://www.una.ac.cr/educare

CORREO: educare@una.cr

o ciega o alguno en silla de ruedas, todos los casos son distintos y hay muchas gamas" (P18). En otros casos algunas personas docentes no poseían respuestas claras ante la situación planteada como: "los contenidos se manejan tal cual para todos los estudiantes" (P8), "Pero me acerqué al grupo para solicitarles colaboración en la parte oral" (P19).

Sin embargo, este nuevo reto no los detiene en la búsqueda de posibles soluciones que simplifiquen los escenarios educativos para esta población. El personal docente busca diversas estrategias que impacten positivamente el aprendizaje asegurando oportunidades al tiempo que cada docente define los mínimos necesarios para atender lo mejor posible al estudiantado, así como los requerimientos del contexto social (Blanco, 2008; Fernández, 2012).

Así pues, a pesar de los avances en la inclusión educativa mediante esfuerzos realizados por las universidades, aún se requiere el establecimiento de líneas de acción para el seguimiento de los procesos educativos del estudiantado con discapacidad sensorial que permitan la identificación y abordaje desde la inscripción en la universidad hasta su egreso. De igual forma, se reitera que las personas docentes como actoras del acto pedagógico, juegan un papel fundamental dentro del currículo debido a que, en conjunto con el estudiantado, son quienes logran generar procesos equitativos e incluyentes; por tanto, las capacitación en torno al tema debe ser prioridad tanto para docentes con estudiantes con NEl, así como para la comunidad en general.

\section{Categoría 2. Permanencia de estudiantes con discapacidad sensorial}

\section{Subcategoría 4. Planificación de la enseñanza}

Esta etapa del proceso educativo genera en el personal docente diversas miradas respecto a la atención de la población con discapacidad sensorial que van desde el cumplimiento de lo establecido al afirmar: "no existe una información previa, que le permita al docente conocer la existencia de un estudiante con discapacidad, y eso no permite la planeación metodológica de acuerdo a las necesidades" (P15); hasta la improvisación mediada por el diálogo y acompañamiento constante por docentes o bien, un compañero o compañera de curso: "sigo el plan inicial, igual yo estoy pendiente de todos para hacer las correcciones, mis clases son las bases y ya de ahí empezamos es a experimentar a jugar con más ingredientes, pero siempre son más o menos las mismas técnicas, entonces es la base que él ya tiene" (P9), "los aprendizajes son diferentes, miro las dificultades y trato de acercarme a cada uno" (P10), "Ideal hacer una mediación y una reafirmación del aprendizaje a nivel individual" (P16).

Es claro que los educadores y las educadoras demuestran buena voluntad a la hora de formar estudiantes con discapacidad, pero más allá de lo anterior, deben tener claro que esto es un derecho fundamental, por tanto, debe ser indispensable que conozcan los problemas que atraviesa el estudiantado. Coll (1991) plantea que un sistema educativo debe encaminarse al estudio de las prácticas educativas en el ámbito interdisciplinar del aula de clase, como un 
medio de organización y políticas que medien el proceso de enseñanza-aprendizaje, por tanto, se entiende que la relación docente-estudiante debe ser horizontal, y no una relación de poder. En este caso, reconocer los diferentes canales de comunicación propios para este estudiantado y aplicar las opciones como las tecnologías asistidas que representan un avance significativo en el acceso a la información, a la comunicación y al conocimiento, para con esto dar fluidez a las clases.

\section{Subcategoría 5. Rutinas de clase}

En la pregunta guía realizada al personal docente acerca de cómo desarrollan sus clases, surgieron las respuestas presentadas a continuación: "realizo la planeación preestablecida del tema correspondiente y la estrategia a utilizar", "generalmente, para enseñar inglés siempre hay un protocolo, una actividad de calentamiento, y luego si se introduce el tema, que pueden ser lecturas, análisis de frases, luego el análisis del vocabulario y estructura, trabajo en equipo y por último la parte individual", "pregunto qué fue lo que dejamos de tarea para esa clase, escuchamos las respuestas de varias personas, se repite un poquito lo que se dijo en la clase anterior y empieza la clase nueva".

Por el contrario, otras personas docentes no tienen como tal una rutina pre-elaborada, "Mis clases no son teóricas a mí me gusta variar, que el estudiante, participe, que pase al tablero, que interactúe conmigo, que traiga sus propios problemas, uso las TIC de información para preparar mis clases, posteo clases en la página", "es exactamente el diálogo, interactuar con el estudiante y conocer las pequeñas dificultades para que ellos se sientan muy bien y lo solucionen, que el aprendizaje les llegue", "al inicio yo les digo cómo vamos a repartir el tiempo, por lo general mis clases no son magistrales porque me gusta es que estén haciendo talleres, que se muevan".

\section{Subcategoría 6. Estrategias pedagógicas}

En la educación inclusiva, el momento con más dificultados para docentes se reduce a las estrategias pedagógicas que debe emplear de manera que se dé a entender y, especialmente, el estudiantado con discapacidad logre desarrollar las habilidades requeridas, sin afectar el proceso de sus pares. De ahí que la claridad de los objetivos de aprendizaje para esta población y rutinas claras implementadas por el docente con apoyos razonables, favorecen significativamente los procesos de aprendizaje. Los docentes y las docentes entrevistadas afirman:

"trabajo la parte didáctica, como con juego de roles y desarrollo de casos" (P15), "También es conveniente enviar los textos con antelación para que el estudiante sordo tenga el tiempo suficiente de mirar cuales son las palabras desconocidas para él del español, las ubique, las contextualice y luego las pueda relacionar en clase (...) Ya sé que cuando escribo en el tablero tengo que hacerlo de otro tamaño, con un marcador que sea visible para ella. Como sé que ella está ahí, ya sé que la instrucción debe ser muy clara y precisa" (P16). 
doi: http://dx.doi.org/10.15359/ree.21-3.15

URL: http://www.una.ac.cr/educare

CORREO: educare@una.cr

Tener claridad sobre a quién se dirige la clase permite identificar acciones que promuevan la atención escolar dentro del aula, al tiempo que se reconoce a cada estudiante con discapacidad como un sujeto social y de derechos. De igual manera, estas acciones permiten al estudiantado avanzar no solo en la adquisición de conocimiento sino también en el camino laboral. Al respecto, Meirieu (2005, citado por Anijovich y Mora, 2010) considera:

Crear rutinas en relación con el trabajo preciso que implica -constancia; respeto de horarios y espacios; articulación entre tiempos, espacios, actividades y participantesequivale a realizar un ejercicio de socialización y democracia. El desafío está en pasar de la rutina impuesta por el docente, progresivamente, a las rutinas creadas por los alumnos para sí mismos. Cuando uno es dueño de su propia rutina, tiene también que aprender a probarla, a observar si funciona, a modificarla y a comprometerse con el cumplimiento de ella. (p. 29)

Desdeuna visión educativa, laatención deestapoblación medianteestrategias pedagógicas que atiendan y orienten las habilidades y competencias de estudiantes con discapacidad es una manera de materializar el enfoque social de la discapacidad. La emancipación ocupa el rol central y promueve en cada estudiante las oportunidades para participar en la sociedad como un actor social promotor del desarrollo local (Coll, 1991; Victoria, 2013).

Se resalta que la mayoría de las personas docentes implementan estrategias pedagógicas tanto por convicción personal, como por directrices de la universidad, en la búsqueda de brindar mayor calidad en la educación que se imparte. No obstante, se hace imperiosa la necesidad de formar a las personas docentes en estrategias inclusivas que les permitan hacer una planeación centrada en la persona, en sus potencialidades y capacidades, dejando de lado su discapacidad.

\section{Subcategoría 7. Materiales didácticos}

A lo largo de este recorrido, se evidenció que existe un compromiso en la labor pedagógica orientada a la inclusión educativa de estudiantes con discapacidad; no obstante, la mayoría de docentes desconoce materiales especializados en los diferentes recursos didácticos que facilitarían esta labor; no obstante, una parte no se limita y promueve acciones flexibles para corroborar el aprendizaje en esta población (MEN, 2007).

Algunas personas docentes expresaron: "utilizo material didáctico virtual ubicado en Tema"(P7); la mayoría referenció no utilizar material didáctico en sus clases, "ninguno, la clase es muy dinámica y hay mucha oralidad" (P10); por ende, es apremiante que las universidades, como instituciones garantes de derechos implementen procesos tecnológicos y capaciten al personal docente sobre el manejo de estos elementos, para que se posibilite el acceso a la formación del estudiantado con discapacidad. 


\section{Subcategoría 8. Evaluación}

La forma de evaluación del personal docente a sus estudiantes con discapacidad es diversa, pero con la misma rigurosidad y exigencia de los demás compañeros o compañeras: "No la excluyo, ella sabecuándo tienequeexponeryyo prestomiatención parapodercaptarloquemeestádiciendo"(P9). Sin embargo, algunas personas docentes hacen distinciones al momento de evaluar a estudiantes con discapacidad, "No puede ser igual a la de los demás, porque no es su idioma, están en otro nivel, el sordo sería capaz siempre y cuando tenga claro el concepto y lo que se le pregunta, tendría que haber una retroalimentación de la pregunta en caso de que el sordo por sí solo no le encuentre la claridad" (P6).

De lo anterior, se destaca la actitud positiva que tiene el personal docente frente al estudiantado con discapacidad y a su proceso. No obstante, aún se desconoce que un estudiante con discapacidad necesita, en la mayoría de ocasiones, el doble del tiempo para desarrollar una actividad, una lectura o un trabajo, pues si no cuenta con apoyos desde el inicio de su formación, muy seguramente tendrá problemas cuando los conocimientos sean más complejos. Por tanto, Martínez y Rumie (2012) establecen los instrumentos tecnológicos como recursos para evaluar al estudiantado con NEEl:

Se plantea que la evaluación responda a la diversidad poblacional ... y sirva, además de medir las competencias de los estudiantes, para fomentar la reflexión sobre la práctica pedagógica. Este proceso evaluativo permite y ayuda al docente a planificar sus labores y proveer elementos que disminuyan las barreras para el aprendizaje y propicien la participación de todos, a partir del conocimiento de las características de cada estudiante. (p. 6)

Es decir, en términos de un enfoque social de la discapacidad, el derecho a la educación es el derecho a aprender a lo largo de la vida. Concebir la educación como derecho, y no como mero servicio o mercancía implica que las instituciones de educación superior deben respetar, garantizar, proteger y promover este derecho desde diversas formas de evaluación que no sean las tradicionales, por cuanto su violación vulnera el ejercicio de otros derechos humanos.

\section{Subcategoría 9. Experiencias significativas}

En la entrevista se le pidió al personal docente compartir una experiencia en su labor educativa, a saber:

"para míel punto de partiday referente es la prueba diagnóstica, con eso empiezo a revisar a nivel particular cuáles son las dificultades y empiezo a trabajar con talleres de acuerdo a la dificultad con respecto al grupo" (P20); "A mí me gusta trabajar mucho con el aprendizaje colaborativo, y también trabajo algo de aprendizaje significativo; mirar el contexto", "en el caso de los estudiantes con discapacidad, también creo que con ellos es importante trabajar la parte personalizada, las tutorías, porque en clase es difíil sentarme solamente con un estudiante" (P13) 
doi: http://dx.doi.org/10.15359/ree.21-3.15

URL: http://www.una.ac.cr/educare

CORREO: educare@una.cr

De acuerdo con lo explicado por el personal docente, es claro que el ejercicio pedagógico cambia cuando en el grupo hay estudiantes con discapacidad, lo cual supone para el maestro o maestra nuevas formas de ver y pensar la educación. No obstante, para que estas experiencias sean cada día más exitosas y significativas se debe dar un nuevo enfoque al rol docente como una persona coequipera que sea capaz de transformar su quehacer pedagógico y cerrar la brecha que existe entre la educación tradicional y la educación inclusiva

De acuerdo con lo anterior, Sánchez (2011) expresa:

Se han iniciado distintas experiencias que suponen un reto para la institución universitaria, aunque no resultan suficientes debido a que nos encontramos ante una demanda social dinamizada por la integración en los diferentes niveles del sistema educativo y que exige la normalización de la integración en la universidad. (p. 577)

En otras palabras, la educación como bien común y un derecho humano fundamental exige que esta sea de calidad, promoviendo el máximo desarrollo de las múltiples potencialidades de cada persona. Avanzar hacia sistemas educativos más inclusivos requiere una firme voluntad política que se refleje en el desarrollo de políticas de largo plazo que involucren a los diferentes sectores del gobierno y a la sociedad civil. Desde la educación inclusiva, se requiere un equilibrio entre excelencia y equidad con la finalidad que el alumnado con NEE aprendan lo necesario para el desarrollo de su proyecto de vida personal y familiar.

\section{Subcategoría 10. Discriminación}

Mediante la indagación a los docentes y las docentes sobre el manejo del aula de clase frente a la aceptación o discriminación hacia el estudiantado con discapacidad por parte de sus pares $y$, algunos educadores o educadoras, no respondieron claramente, aunque hicieron varias apreciaciones al respecto:

"Normal, aunque él a veces se aísla ... yo noto es cierto grado de intolerancia de algunos de los compañeros de la estudiante hacia su situación" (P17); "no, en mi curso no, y de hecho los compañeros la ayudan, siempre están muy pendientes de ella" (P14).

Respecto a lo referido por el personal docente, sobre las dinámicas de aula, si bien es cierto que algunas personas con discapacidad tienden a aislarse, porque a veces el medio se vuelve hostil o no se sienten parte del grupo; se hace necesario generar espacios de sensibilización y toma de conciencia frente a la diversidad educativa en las universidades. Al respecto de esto Moreno, Rodríguez, Saldaña y Aguilera (2006) afirman: 
Si las actitudes de los no discapacitados ante las personas con discapacidad es un asunto con consecuencias importantes en la integración social de las personas con algún tipo de problema en su desarrollo cognitivo, sensorial o de cualquier otro tipo, más lo es cuando los "no discapacitados" son, precisamente, aquellos profesionales que han de velar por la rehabilitación de estas personas, por su integración social, educativa y laboral y, en definitiva, por que [sic] se vean satisfechas sus necesidades especiales. (p. 1)

Estudiantes y docentes manifestaron durante el proceso de investigación que es necesaria mayor capacitación en cómo manejar el aula y apoyar al estudiantado con discapacidad, debido a que no todas las discapacidades son iguales y unas requieren más apoyo que otras. De ahí la importancia no solo de capacitar para atender la situación a nivel micro - desde el aula de clase-; sino el diseño de programas de apoyo y seguimiento a la labor docente para atender la situación a nivel macro - desde el exterior del aula de clase-.

\section{Conclusiones y recomendaciones}

De acuerdo con los resultados, se concluye que, aunque el personal docente realiza esfuerzos significativos para atender las necesidades de estudiantes con discapacidad, las universidades no cuentan con una ruta de atención institucional para estos grupos. De esta manera, se cuenta con barreras que dificultan todo proceso de inclusión escolar así como procesos básicos al sistema educativo: un ingreso adecuado y personalizado que permita orientarse y la adquisición de habilidades para el ingreso al mundo laboral.

Es vital que los cuerpos docentes desarrollen estrategias educativas y pedagógicas inclusivas, acordes al modelo de desarrollo humano y social que exige afianzar en unas competencias desde el ser, saber y hacer de acuerdo con las necesidades de las personas. En este sentido, desde el interior de cada programa, escuela o facultad, se deben establecer lineamientos donde el objetivo de cada programa académico deberá ser la equiparación de oportunidades mediante la flexibilización de dinámicas de clase y evaluación, en este caso, para el estudiantado con discapacidad sensorial.

En las universidades se debe plantear la adaptación o flexibilización del currículo y sus contenidos temáticos de acuerdo con los programas de formación académica y la realidad del estudiantado en formación, en el caso que compete a este estudio la discapacidad visual o auditiva. Se requiere diseñar y establecer programas de tutorías personalizadas a estudiantes con discapacidad cuya función será asesorar en la profundización de las temáticas complejas, en articulación con cada docente, siempre y cuando el estudiante o la estudiante lo solicite en el departamento especializado de cada una de las universidades. 
doi: http://dx.doi.org/10.15359/ree.21-3.15

URL: http://www.una.ac.cr/educare

CORREO: educare@una.cr

De igual manera, se resalta que las universidades no niegan el derecho a la educación, pero la permanencia y promoción del estudiantado a lo largo del desarrollo de la carrera no están garantizadas. Un factor de éxito que contribuye en la promoción del estudiantado con discapacidad es conocer, de manera anticipada, cuál va a ser el desarrollo de las clases, rutinas, ejercicios, trabajos, dinámicas de clase, así como previamente tener el material adecuado a su necesidad: el lenguaje que se requiera, el tamaño adecuado, o el medio pertinente ;así como la noción de la temática que se va a tratar, los autores y autoras que se van a estudiar, los sitios web donde se puede ampliar la información; de tal forma que se posibilite la apropiación del conocimiento.

Como se evidenció a lo largo del documento, docentes de las instituciones objeto de estudio carecen de capacitaciones en didácticas específicas; no obstante, han realizado acciones significativas que atienden y reconocen al estudiantado con discapacidad como un sujeto de educación y futuro actor social. Por tanto, es imperante que este segmento poblacional y sus docentes generen intercambio de experiencias: en primer lugar, con el personal docente que recibirá a estudiantes con discapacidad, con el fin de dar continuidad al proceso y las estrategias flexibles y; en segundo lugar, otras comunidades educativas, las cuales pueden ser lideradas por un comité institucional, de tal modo que se trabaje de manera articulada para que los diferentes departamentos de las universidades conozcan y promuevan el enfoque de educación inclusiva.

Finalmente, De igual manera, es de carácter imperante contar con profesionales que se hayan formado en lengua de señas y profesionales con experticia en las áreas tiflológicas como, braille, ábaco, orientación, manejo espacial y tifloinformática entre otras; al tiempo que se articule el trabajo participativo con la comunidad educativa que interviene en la realidad social del estudiantado con discapacidad: personal docente y administrativo, y sus pares sin discapacidad.

De ahí que, entre algunos elementos indispensables para alcanzar esta meta se requiere que las universidades piensen en el acceso lingüístico además de herramientas tecnológicas que permitan el acceso a la información (tiflotecnológicos). Así pues, algunos proyectos de grado de programas como Ingeniería de Sistemas y los programas de las diversas licenciaturas de las universidades podrían estar orientados a desarrollar los programas mencionados que favorezcan la gestión de la información para esta población, así como investigación educativa en didácticas flexibles que apoyen la labor docente.

\section{Referencias}

Ainscow, M. (2001). Desarrollo de escuelas inclusivas. Ideas, propuestas y experiencias para mejorar las instituciones escolares. Madrid: Narcea.

Andrés, M. D. y Sarto, M. P. (2009). Escuela inclusiva: Valores, acogida y convivencia. En M. P. Sarto y M. E. Venegas (Coords.), Aspectos claves de la educación inclusiva (pp. 85-118). Salamanca: Publicaciones del Inicio. Recuperado de http://sid.usal.es/idocs/F8/FDO22224/educacioninclusiva.pdf 
Anijovich, R. y Mora, S. (2010). Estrategias de enseñanza. Otra mirada al que hacer en el aula. Buenos Aires: Aique Grupo Editor.

Asamblea Nacional Constituyente. (4 de julio de 1991). Constitución Política de Colombia (2ª ed.). Colombia: Editorial Legis.

Barton, L. y Armstrong, F. (2001). Disability, education and inclusion: Cross-cultural issues and dilemmas. En G. L. Albrecht, K. D. Seelman y M. Bury (Eds.), Handbook of Disability Studies (pp. 693-710). SAGE Publications. doi: https://doi.org/10.4135/9781412976251.n31

Bayliss, P., Van Hove, G., Windels, D., Carro, I., \& Hinz, A. (1997). Manifiesto of the Carrot Scoup Group: Inclusion and Integration. The European Electronic Journal on Inclusive Education in Europe, 2.

Blanco, R. (2008). Marco conceptual sobre educación inclusiva. En Unesco (Ed.), La educación inclusiva: El camino hacia el futuro (pp. 5-14). Ginebra: Unesco. Recuperado de http:// www.ibe.unesco.org/fileadmin/user upload/Policy Dialogue/48th ICE/CONFINTED 48 Inf 2 Spanish.pdf

Coll, C. (1991). Psicología y curriculum. Una aproximación psicopedagógica a la elaboración del curriculum escolar. Barcelona: Paidós.

Coll, C. (1996). Psicopedagogía: Confluencia disciplinar y espacio profesional. En C. Monereo e I. Solé (Coords.), El asesoramiento psicopedagógico: Una perspectiva profesional y constructivista. Psicología y Educación (pp. 33-52). Madrid: Alianza Editorial.

Congreso de Colombia (28 de diciembre de 1992). Ley 30 de 1992: por el cual se organiza el servicio público de la educación superior. Bogotá: Autor. Recuperado de http://www.mineducacion. gov.co/1621/articles-85860 archivo pdf.pdf

Congreso de Colombia (27 de febrero de 2013). Ley Estatutaria 1618 de 2013: Por medio de la cual se establecen las disposiciones para garantizar el pleno ejercicio de los derechos de las personas con discapacidad. Bogotá: Congreso de Colombia. Recuperado de http://wsp. presidencia.gov.co/Normativa/Leyes/Documents/2013/LEY\%201618\%20DEL\%2027\%20 DE\%20FEBRERO\%20DE\%202013.pdf

De la Oliva, D. (2007). Guía para la evaluación y mejora de la educación inclusiva. México: UIC. Manuscrito inédito.

Fernández J. M. (2012). Capacidades y competencias docentes para la inclusión del alumnado en la educación superior. Revista de la Educación Superior, 41(162), 9-24. Recuperado de http://publicaciones.anuies.mx/pdfs/revista/Revista162 S1A1ES.pdf 
doi: http://dx.doi.org/10.15359/ree.21-3.15

URL: http://www.una.ac.cr/educare

CORREO: educare@una.cr

Freire, P. (2004). Pedagogía de la autonomía. Saberes necesarios para la práctica educativa. Sao Paulo: Paz e Terra.

Gutiérrez, M., Martín, M. V. y Jenaro, C. (2014). El index para la inclusión: Presencia, aprendizaje y participación. Revista nacional e internacional de educación inclusiva, 7(3), 186201. Recuperado de http://www.revistaeducacioninclusiva.es/index.php/REl/article/ view/139/133

Hernández, R., Fernández C., Baptista, P. (2010). Metodología de la investigación (3ª ed.). México: McGraw Hill.

Latorre, A., Del Rincón, D. y Arnal, J. (1996). Bases metodológicas de la investigación educativa. Barcelona: Labor.

Martínez, A. y Rumie, S. M. (2012). Pedagogía en valores: Una estrategia de apropiación del proyecto de formación inclusiva, en los docentes y estudiantes de los grados preescolar y primaria, de la jornada matinal de la institución educativa Olga González Arraut. Primer semestre 2012. Universidad de Cartagena. Colombia, Cartagena de Indidas. Recuperado de $\quad$ http://190.242.62.234:8080/jspui/bitstream/11227/3403/1/Py\%20Pedagogia\%20 en\%20valores final.pdf

Martínez, P.C. (2006). El método de estudio de caso: Estrategia metodológica de la investigación científica. Pensamiento \& Gestión, 20, 165-193. Recuperado de http://www.redalyc.org/ pdf/646/64602005.pdf

Ministerio de Educación Nacional (MEN). (1 de septiembre al 31 de diciembre de 2007). Educación para todos. Altablero No. 43. Recuperado de http://www.mineducacion.gov. co/1621/article-141881.html

Ministerio de Educación Nacional (MEN). (1 de enero al 31 de marzo de 2008). Evaluación para la inclusión. Altablero No. 44. Recuperado de http://www.mineducacion.gov.co/1621/ article-162382.html

Moreno, F. J., Rodríguez, I. R., Saldaña, D. y Aguilera, A. (2006). Actitudes ante la discapacidad en el alumnado universitario matriculado en materias afines. Revista lberoamericana de Educación, 40(5), 1-12. Recuperado de http://rieoei.org/investigacion/1491Moreno.pdf

Organización Mundial de la Salud (OMS). (2001). Clasificación internacional del funcionamiento, de la discapacidad y de la salud. Ginebra: Autor. Recuperado de https://aspace.org/assets/ uploads/publicaciones/e74e4-cif 2001.pdf

Ortiz, M. (2000). Hacia una educación inclusiva. La educación especial ayer, hoy y mañana. Revista Siglo Cero, 31(187), 5-11. 
Palacios, A. (2008). El modelo social de discapacidad: Orígenes, caracterización y plasmación en la Convención Internacional sobre los Derechos de las Personas con Discapacidad. Madrid: Ediciones Cinca. Recuperado de http://www.tiempodelosderechos.es/docs/jun10/m6.pdf

Rodríguez, S. y Ferreira, M. A. V. (2010). Desde la dis-capacidad hacia la diversidad funcional: Un ejercicio de dis-normalización. Revista Internacional de Sociología, 68(2), 289-309. doi: https://doi.org/10.3989/ris.2008.05.22

Rosano, S. (2007). La cultura de la diversidad y la educación inclusiva. Recuperado de http://benu. edu.mx/wp-content/uploads/2015/03/La cultura de la diversidad y la educacion inclusiva.pdf

Sánchez, A. (2011). La Universidad de Almería ante la integración educativa y social de los estudiantes con discapacidad: Ideas y actitudes del personal docente e investigador. Revista de Educación, 354, 575-603. Recuperado de http://www.revistaeducacion. educacion.es/re354/re354 23.pdf

Sandoval, C. A. (1996). Investigación cualitativa. Bogotá: ICFES. Recuperado de http://panel. inkuba.com/sites/2/archivos/manual\%20colombia\%20cualitativo.pdf

Stake, R. E. (1998). Investigación con estudio de casos. Madrid: Ediciones Morata. Recuperado de http://investigacionsocial.sociales.uba.ar/files/2013/03/STAKE investigacion-conestudio-de-casos.pdf

Vargas, J. y Calvo, G. (1987). Seis modelos alternativos de investigación documental para el desarrollo de la práctica universitaria en educación: El caso del proyecto de extensión REDUC - Colombia en la Universidad Pedagógica Nacional. Revista Educación Superior y Desarrollo, 5.

Vásquez, A y Cáceres, N. (Eds.). (2008). El abordaje de la discapacidad desde la atención primaria de la salud. Buenos Aires: Organización Panamericana de la Salud. Recuperado de http:// www.paho.org/arg/publicaciones/otras\%20pub/discapacidad.pdf.

Velázquez, E. (2010). La importancia de la organización escolar para el desarrollo de escuelas inclusivas (Tesis doctoral). Universidad de Salamanca, España. Recuperada de http:// gredos.usal.es/jspui/bitstream/10366/76601/1/DDOMI Velazquez Barragan E Laimportanciadlaorganizacionescolar.pdf

Victoria, J. A. (2013). El modelo social de la discapacidad: Una cuestión de derechos humanos. Boletín mexicano de derecho comparado, 46(138), 1093-1109. Recuperado de http://www. scielo.org.mx/pdf/bmdc/v46n138/v46n138a8.pdf 
doi: http://dx.doi.org/10.15359/ree.21-3.15

URL: http://www.una.ac.cr/educare

CORREO: educare@una.cr

Zaccagnini, M. (2008). Configuraciones del sujeto pedagógico contemporáneo. Intersecciones; tensiones y fracturas; continidades y discontinuidades, entre la epistemología de las prácticas educativas escolares y la realidad social. Grupo G.I.S.E.A. Universidad Nacional de Mar del Plata. Argentina.

Zárate, R., y Rodríguez, D. A. (2014). Los derechos de las personas en situación de discapacidad: Una respuesta desde la responsabilidad social. Revista Eleuthera, 10, 38-57. Recuperado de http://vip.ucaldas.edu.co/eleuthera/downloads/Eleuthera10 4.pdf 